\title{
Check plots in field breeding experiments
}

\author{
Stanisław Mejza, Iwona Mejza \\ Department of Mathematical and Statistical Methods, Poznań University of Life Sciences, \\ Poland, e-mail: smejza@up.poznan.pl,imejza@up.poznan.pl
}

\begin{abstract}
SUMMARY
This paper deals with the problems of selection in the early stages of a breeding program. During the improvement process, it is not possible to use an experimental design that satisfies the requirement of replicating all the treatments, because of the large number of genotypes involved, the small amount of seed and the low availability of resources. Hence unreplicated designs are used. To control the real or potential heterogeneity of experimental units, control (check) plots are arranged in the trial.

There are many methods of using the information resulting from check plots. All of the usually applied adjusting methods for unreplicated experiments are appropriate for some specific structure of soil fertility. Their disadvantage is the fact that, before and also after the experiment, we usually do not know what a kind of soil structure is present in the experiment. Hence we cannot say which of the existing methods is appropriate for a given experimental situation. The method of inference presented below avoids this disadvantage. It is always appropriate, because of the fact that a trend of soil variability is identified and estimated. In the paper the main tool used to explore this information will be based on a response surface methodology. To begin with we will try to identify a response surface characterizing the experimental environments. We assume that observed yield (or another trait) results directly from two components, one of them due to soil fertility and the other due to the genotype effect. This means that difference between observed yield and forecast can be treated as the estimate of a genotype effect. The obtained response surface will then be used to adjust the observations for genotypes. Finally, the data so adjusted are used for inferences concerning the next stage of the breeding program. The theoretical considerations are illustrated with an example involving yields of spring barley.
\end{abstract}

Key words: breeding field experiments, check plots, phenotypic selection, unreplicated experiments.

\section{Introduction}

This work deals with selection experiments in the early stages of breeding programs. In the early stages of plant breeding trials, it is often difficult to use 
an experimental design that satisfies, for example, Fisher's principles. This is connected with the number of genotypes (experimental objects) evaluated, which usually ranges from several hundred to thousands. The breeder is mainly interested in the selection and identification of superior genotypes for further breeding. Additionally to the very large number of genotypes (lines) to be evaluated, these experiments often have a limitation resulting from the small amount of seeds and the low availability of resources. Thus replication may not always be possible, especially if the plots are to be large enough for proper yield assessment (Kempton 1984). Consequently, in this case unreplicated experiments are commonly adopted. Then, to control the real or potential heterogeneity of experimental units (field plots), replicated check variety plots are usually distributed over the field experiment area. Control plots are only effective if the yield (or another trait) of the checks shows the same general pattern of response across the trial as the test genotypes. Otherwise, the adjustment of test plot yields by the yields of the checks may lead to wrong estimation of genotype effects and finally to a wrong selection. This means that the arrangements and frequencies of check plots play a crucial role in selecting genotypes in such experiments.

Different random and systematic arrangements of check plots have been used (see for example Kempton 1984, Sebolai et al. 2005, Cullis et al. 1989, Baker and McKenzie 1967). Moreover, inference methods for unreplicated breeding trials are proposed, for example, by Cullis et al. 1989, Kempton and Fox 1997. The latter additionally conducted a large simulation study to compare adjustments by the methods of spatial analysis, moving averages and check plots. The variability of units with their geometrical structure in the experiment is used by the proposed methods to adjust the average values of observed characteristics on genotypes. More exactly, the yields of the check plot variety are used as the yardstick against which to assess the yield of each test (genotype) plot.

The simplest experimental designs are based on a systematic arrangement of equally spaced checks. Often the check plots are used to construct an 
environmental index. The variability of units with their geometrical structure in the experiment is used by the proposed methods to adjust the average values of observed characteristics on genotypes. Usually, to construct the environmental index, we use, for example, the yield from the nearest check plots, the mean yield of the two nearest checks, one on either side of the test plot, the weighted mean of those two checks, where weights are inversely related to the distance from the genotype plots (in all directions), etc. Also, there are many other suggestions as to how to use check plots, boarding check plots, to estimate the spatial variability of environment. Recently the method called "moving average" has frequently been recommended for use (see for example Utz 1997).

All of the usually applied adjustment methods for unreplicated experiments are appropriate for some specific structure of soil (environment) fertility. Their disadvantage is the fact that, before and also after the experiment, we do not know what kind of soil (environmental) structure is present in the experiment. Hence we cannot say which of the existing methods is appropriate to a given experimental situation. The method of inference given in this paper avoids this disadvantage. It is always appropriate for any structure of soil (environment). This results from the fact that we estimate the environmental structure independently for each experiment. The problem which arises concerns only the quality of the response surface estimated in the experiment. To choose the proper response surface estimate, any selection criteria for linear or nonlinear models may be used.

\section{Method}

In this paper we propose a new approach to the design and analysis of unreplicated breeding experiments. The density and arrangement of check plots play the main role.

Let us assume that the experimental units have a row-column structure. We denote by $(i, j)$ the coordinate of the check plot center. Let us note that the same genotype occurs on the check plots. The environment (soil variability) is the 
main source of the variability of observations. Then the yield on the check plot can be expressed as:

$$
\mathrm{Y}_{i j}=\mathrm{F}(i, j)+\mathrm{e}_{i j}, \mathrm{E}\left(\mathrm{Y}_{i j}\right)=\mathrm{F}(i, j),
$$

where $\mathrm{F}(i, j)$ is some response surface function, while $\mathrm{e}_{i j}$ denotes an experimental error.

The response surface function $\mathrm{F}(i, j)$ characterizes the true, real environmental variability (for example variation in soil fertility). In fact the function $\mathrm{F}(i, j)$ describes the yield response of the check plot genotype over the experimental plots. In genotype selection we do not need to consider the genotype that occurs on the check plot.

This results from the fact that the same genotype occurs on the check plots. In the selection problem we are not interested in making a ranking with respect to the check plot object, but with respect to the test genotypes. Hence we can treat the function $\mathrm{F}(i, j)$ as in fact describing the true, real environmental fertility and variability.

As usual we assume that random terms $\mathrm{e}_{i j}$ are independently and identically normally distributed over all experimental units (not only over check plots) with expected value equal to 0 and common variance equal to $\sigma^{2}$. We can write this in short as $\mathrm{e}_{i j} \sim \mathrm{N}\left(0, \sigma^{2}\right)$. Additionally all covariances are equal to 0 . The form of the function $\mathrm{F}(i, j)$ is unknown and it is necessary to estimate it.

Let $F(i, j)$ be the estimate of the function $\mathrm{F}(i, j)$. To estimate the response surface we can use any relevant available statistical tools (see for example: Box and Draper 2007, Bradley 2007, Khuri and Cornell 1987, Khuri 2006, Myers and Montgomery 2001).

Let us assume that the estimate $F(i, j)$ obtained is acceptable and sufficiently explains the environmental response over the experimental material (an experimental field). Additionally, based on the check plot observations, we estimate the common variance $\sigma^{2}$. We assume that this estimate is valid for all genotype plots. 
In the next step we generate a forecast for all plots with genotypes. Let $\hat{F}(p, q)$ denote the forecast of the response on the plot $(p, q)$ (for genotype occurring on this plot). Then the difference

$$
\mathrm{Y}_{p q}-\hat{F}(p, q)=\hat{G}(p, q)
$$

can be treated as the estimate of the effect of genotype $\mathrm{G}(p, q)$ occurring on the plot $(p, q)$, where as previously $\mathrm{Y}_{p q}$ denotes the observation obtained for the genotype occurring on the plot $(p, q)$. In the case of unreplicated experiments it is impossible to separate the environmental effect from the genotype effect.

Finally we have the estimate of the genotype effects and estimate of the error variance MSE $=\hat{\sigma}^{2}$. These facts can be used to build a ranking (selection) of the genotypes. There are many ways to build the ranking of genotypes. Here we suggest the use of some test for grouping genotypes. We propose to apply a simultaneous test procedure, such as Tukey's test or the approximated (Bonferroni) t-test.

The hypotheses to be tested can be expressed as:

$$
\begin{aligned}
& H_{0}: G(p, q)-G\left(p^{\prime}, q^{\prime}\right)=0 \quad \text { forall }(p, q),\left(p^{\prime}, q^{\prime}\right), p \neq q, p^{\prime} \neq q^{\prime}, p \neq p^{\prime}, q \neq q^{\prime} \\
& H_{1}: G(p, q)-G\left(p^{\prime}, q^{\prime}\right)>0
\end{aligned}
$$

Two additional issues should be discussed. The first concerns check plot arrangements, and the second concerns check plot frequency (see for example Mejza and Marczyńska 2011).

Traditional arrangements include schemes in which the check plots are arranged, for example, in every third row (Holtsmark and Larsen 1905), in every fifth or sixth row in experimental units laid out in two ways (see example), and some others. Between these rows the analyzed genotypes are arranged. There are no clear arguments for such arrangements. Such supporting points of a design do not make it possible to control environmental heterogeneity over the experimental field. Therefore, we propose a systematic and even arrangement of check plots over the whole experimental area. Such arrangements allow one to estimate the environmental response function more exactly. The location scheme of the supporting points in experiments (check 
plots) has a direct impact on the method of estimation of the response surface function and on its goodness of fit. Also, the density of check plots plays an important role in the selection of an appropriate response function. Using systematic designs we have the following potential and useful arrangements. The scheme depends on the frequency (density) of the check plots that the breeder intends to apply in the experiment. For example, if the breeder decides to use about $50 \%$ of units for check plots, he can use one of the schemes:

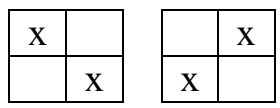

For a density of about $33 \%$ of check plots we can use one of the following potential schemes (from $3 !=6$ ):
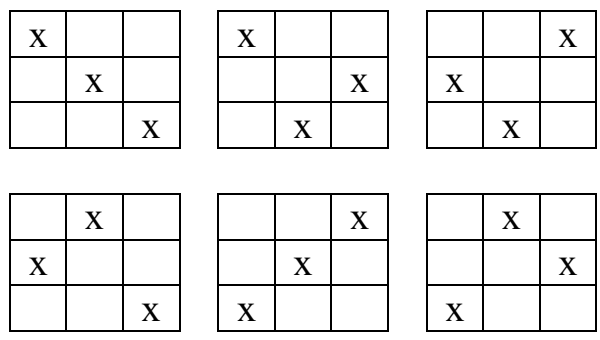

For a density of about $25 \%$ of check plots we can use one of the potential schemes from $4 !=24$. Here we present only four such arrangements.:
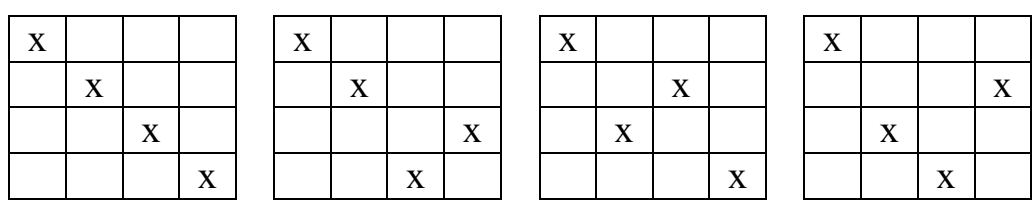

For a frequency of about $20 \%$ of check plots we have $5 !=120$ potential arrangements of check plots. Here we present three of them. 

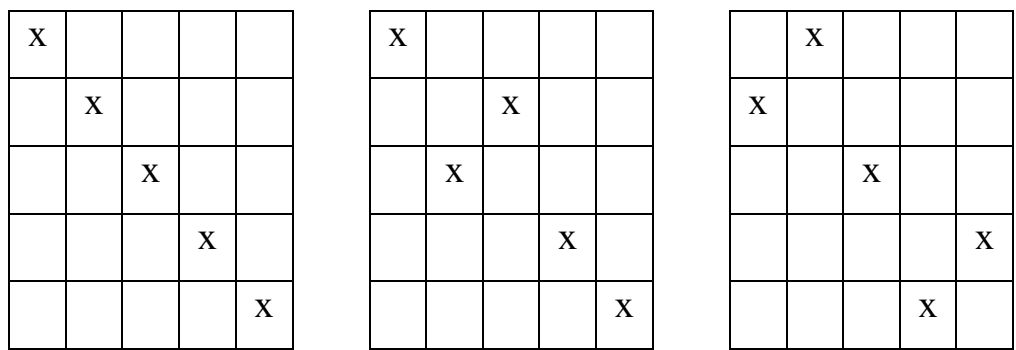

\section{Example}

The above considerations are illustrated with the results obtained for grain yield in unreplicated breeding trials with standards, including 333 lines of spring barley, performed at Modzurów (Poland) plant breeding station. The observed trait in the experiment was the grain yield in $\mathrm{kg}$ per plot. The experiment was conducted in the 2005/2006 season. In the statistical analysis the yields of 333 experimental plots were taken into account. The plots were arranged in 37 rows and 9 columns. In the experiment 63 plots were designated as check plots, and the analyzed genotypes were assigned to 270 plots. The measured (observed) area of the plots was $1 \mathrm{~m} \times 10 \mathrm{~m}$, i.e. $10 \mathrm{~m}^{2}$. The geometrical structure was characterized by $(x, y)$ coordinates with the following values: $x=5,15,25,35$, $45,55,75,85[\mathrm{~m}] ; y=0.5,6.5,12.5,18.5,24.530 .5,36.5[\mathrm{~m}]$. The experimental scheme is given in Table 1. Codes printed in bold and italics denote the check plots (the same genotype). Other codes denote the genotypes (test lines).

The statistical analysis was performed in two stages. At the start we estimate the two-dimensional surface function for check plot yield (marked in bold and italics in Table 1) to characterize the variability of the soil (environment) in the experimental field. Using the methods of model selection, we decided to adopt the estimate of the response surface of the yield in the form: $F(x, y)=4.574+0,160 \mathrm{x}-0.006 \mathrm{x}^{2}+0.0001 \mathrm{x}^{3}+0.227 \mathrm{y}-0.016 \mathrm{y}^{2}+0.0003 \mathrm{y}^{3}+$ $0.0001 \mathrm{xy}$. We reject the hypothesis concerning the adequacy of the model at a significance level of alpha $=0.05$. The coefficient of determination is equal to $74 \%$. The shape of the response surface characterizing the environmental 
Table 1. Scheme of the experiment

\begin{tabular}{|c|c|c|c|c|c|c|c|c|}
\hline 4813 & 4948 & 5069 & 5204 & 5325 & 5460 & 5581 & 5654 & $\mathrm{~J}$ \\
\hline 4814 & 4947 & 070 & 5203 & 326 & 5459 & 5582 & 5653 & 5776 \\
\hline 15 & 4946 & 5071 & 5202 & 5327 & 58 & 33 & 52 & 5777 \\
\hline 4816 & 4945 & 5072 & & 5328 & & & 51 & \\
\hline 4817 & 944 & 5073 & 5200 & 5329 & 54 & 35 & 5650 & 5779 \\
\hline 4818 & 4943 & 5074 & 5199 & 5330 & 5455 & & 49 & 5780 \\
\hline 4819 & 4942 & 5075 & 5198 & 5331 & 5454 & 5587 & 5648 & 5781 \\
\hline 4820 & 4941 & 5076 & 5197 & 5332 & 5453 & 5588 & 5647 & 5782 \\
\hline 4821 & 4940 & 5077 & 5196 & 5333 & 5452 & & & 5783 \\
\hline 4822 & 4939 & 5078 & 5195 & 5334 & 5451 & 5590 & 5645 & 5784 \\
\hline 4823 & 4938 & 5079 & 5194 & 5335 & 5450 & 5591 & 5644 & 5785 \\
\hline 4824 & 4937 & 5080 & 5193 & 36 & 5449 & 92 & 43 & 5786 \\
\hline 4825 & 4936 & 5081 & 5192 & 5337 & 5448 & 5593 & 5642 & 5787 \\
\hline 4826 & 4935 & 5082 & 5191 & 5338 & 5447 & 5594 & 5641 & 5788 \\
\hline 4827 & 4934 & 5083 & 5190 & 5339 & 5 & 5 & 40 & 5789 \\
\hline 4828 & 4933 & 5084 & 39 & 5340 & 45 & 96 & & 5790 \\
\hline 4829 & 4932 & 5085 & 5188 & 5341 & 5444 & 5597 & 5638 & 5791 \\
\hline 4830 & 4931 & 5086 & 5187 & 5342 & 5443 & 5598 & 5637 & 5792 \\
\hline 4831 & 4930 & 5087 & 5186 & 5343 & 5442 & 5599 & 5636 & 5793 \\
\hline 4832 & 4929 & 5088 & 5185 & 5344 & 5441 & 5600 & 5635 & 5794 \\
\hline 4833 & 4928 & 5089 & 5184 & 5345 & 5440 & 5601 & 5634 & 5795 \\
\hline 4834 & 4927 & 5090 & 5183 & 5346 & 5439 & 5602 & 5633 & 5796 \\
\hline 4835 & 4926 & 5091 & 5182 & 5347 & 5438 & 5603 & 5632 & 5797 \\
\hline 4836 & 4925 & 5092 & 5181 & 5348 & 5437 & 5604 & 5631 & 5798 \\
\hline 4837 & 4924 & 5093 & 5180 & 5349 & 5436 & 5605 & 5630 & 5799 \\
\hline 4838 & 4923 & 5094 & 5179 & 5350 & & 5606 & 5629 & 5800 \\
\hline 4839 & 4922 & 5095 & 5178 & 5351 & 5434 & 5607 & 5628 & 5801 \\
\hline 4840 & 4921 & 5096 & 5177 & 5352 & 5433 & 5608 & 5627 & 5802 \\
\hline 4841 & 4920 & 5097 & 5176 & 5353 & 5432 & 5609 & 5626 & 5803 \\
\hline 4842 & 4919 & 5098 & 5175 & 5354 & 5431 & 5610 & 5625 & 5804 \\
\hline 4843 & 4918 & 5099 & 5174 & 5355 & 5430 & 5611 & 5624 & 5805 \\
\hline 4844 & 4917 & 5100 & 5173 & 5356 & 5429 & 5612 & 5623 & 5806 \\
\hline 4845 & 4916 & 5101 & 5172 & 5357 & 5428 & 5613 & 5622 & 5807 \\
\hline 4846 & 4915 & 5102 & 5171 & 5358 & 5427 & 5614 & 5621 & 5808 \\
\hline 4847 & 4914 & 5103 & 5170 & 5359 & 5426 & 5615 & 5620 & 5809 \\
\hline 4848 & 4913 & 5104 & 5169 & 5360 & 5425 & 5616 & 5619 & 5810 \\
\hline 4849 & 4912 & 5105 & 5168 & 5361 & 5424 & 5617 & 5618 & 5811 \\
\hline
\end{tabular}


response (soil fertility) of the experimental field on the basis of check plots is presented in Figure 1.

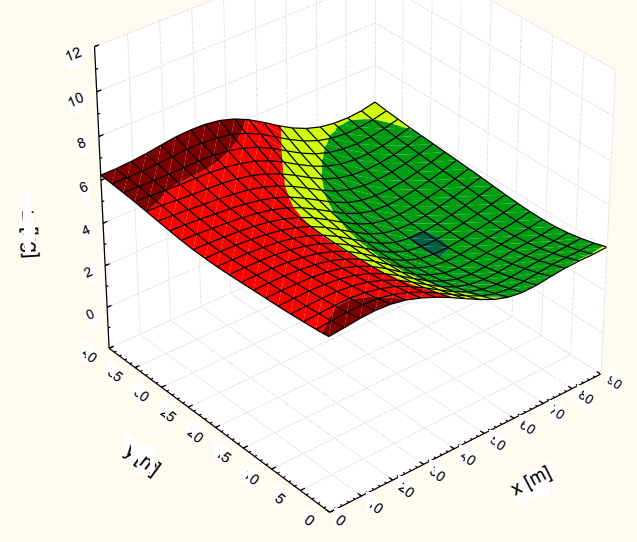

Figure 1. The estimate of the response function for check plots

In the next step we calculate forecasts for all genotypes (all plots with test genotypes). The coordinates of the genotype plot were the central points of the experimental fields. This means that the distances in one direction were 5, 10, $15, \ldots, 85[\mathrm{~m}]$ while those in the second were $0.5,1.5,2.5, \ldots, 36.5[\mathrm{~m}]$.

As a result of analysis of variance performed for check plot yield, we obtain $\mathrm{LSD}_{0.05}=3.82$ for Tukey's test. The ranking of the lines is presented in Table 2, ordered from the genotype with the smallest yield to the largest. This means that the genotype coded 4844 is the worst, while the genotype coded 5336 is the best. Using Tukey's test we observe that 62 lines (up to code 5334) are of small value. In the case where we skip the line coded 4844, the next 220 genotypes can be considered as being of small value. The best genotype, code 5336, is situated close to a check plot on the field. This means that the forecast can be expected to be very good.

In the sense of Tukey's test the last 44 genotypes (from code 5445 to the end) have almost identical yields. This means that for the next step of selection the last 44 genotypes can be recommended. 
Tabela 2. Genotype ranking

\begin{tabular}{|c|c|c|c|c|c|c|c|c|c|c|c|}
\hline & & & & & & & & & & & \\
\hline 4844 & 1 & 4946 & $-0,548$ & 5612 & -0 & 5190 & 0,254 & 5608 & 0,662 & 40 & 03 \\
\hline 5653 & 85 & & 547 & 5457 & 0 & 801 & & 5194 & & & \\
\hline 5652 & & & & & & & & & & & \\
\hline 56 & 5 & & 519 & & & & & 100 & & & 60 \\
\hline 5651 & 448 & & $-0,489$ & & $-0,068$ & & 302 & 47 & & & 071 \\
\hline 5650 & 076 & & $-0,486$ & & $-0,041$ & & 23 & & 0 & & $\underline{1,099}$ \\
\hline 5778 & 822 & & $-0,484$ & 798 & $-0,036$ & 94 & 27 & 51 & & & $\underline{1,111}$ \\
\hline 5776 & $-1,805$ & 885 & $-0,477$ & 5427 & $-0,007$ & 789 & 0,335 & 804 & 0,683 & & $\underline{1,113}$ \\
\hline 4845 & 1,803 & 814 & $-0,472$ & 4824 & $-0,006$ & 102 & 0,337 & 5441 & 0,699 & 41 & $\underline{1,116}$ \\
\hline 5582 & $-1,799$ & 622 & $-0,471$ & 429 & $-0,005$ & 358 & 0,346 & 5078 & 0,708 & $\underline{43}$ & 1,132 \\
\hline 5326 & $-1,505$ & 86 & $-0,47$ & 788 & 0,01 & 091 & 0,346 & 332 & 0,709 & 74 & $\underline{1,134}$ \\
\hline 5649 & 1,489 & 41 & $-0,451$ & 976 & 0,029 & 4945 & 0,347 & 619 & 0,722 & 44 & , 165 \\
\hline & 48 & 780 & $-0,443$ & 92 & 0,033 & 095 & 0,378 & 103 & 0 & 41 & $\underline{1,183}$ \\
\hline & $36-$ & & -0.422 & 14 & 0,036 & 27 & 0 & 616 & & 32 & , 19 \\
\hline 5172 & & & & & & 45 & 42 & & & & 233 \\
\hline 54 & & & & & & 10 & & & & & 247 \\
\hline & & & & & & & & & & & 255 \\
\hline 56 & & & & & & & & & & & 1,257 \\
\hline &, 2 & & $-0,3$ & 640 & 0 , & & & & & & 1,269 \\
\hline 57 & $-1,1$ & 094 & $-0,3$ & 327 & & & 0 & 38 & & & $\underline{1,278}$ \\
\hline 5101 & $-1,15$ & 772 & $-0,341$ & 348 & 0,102 & 803 & 0,506 & 620 & 0,7 & & $\underline{1,29}$ \\
\hline 564 & $-1,15$ & 34 & $-0,3$ & 323 & 0,1 & 169 & 0,509 & 97 & 0 & & $\underline{1,318}$ \\
\hline 57 & $-1,1$ & & $-0,3$ & 91 & 0,1 & 184 & 0, & 22 & 0 & & 1,357 \\
\hline 4916 & $-1,6$ & & -0, & 7 & 0,1 & & & 95 & 0, & & 359 \\
\hline 56 & $-1,0$ & & 0 & - & & & & 96 & & &, 382 \\
\hline & 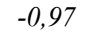 & & & & & & & & & & 1,413 \\
\hline 51 & $-0,9$ & & & & & & & & & & 1,423 \\
\hline & & & & & & & & & & & 429 \\
\hline 5784 & & & & & & & & & & & 1,451 \\
\hline 50 & - & & & & & & & & & & $\underline{1,513}$ \\
\hline 57 & & & & & & & & 46 & & & $\underline{1,52}$ \\
\hline 49 & -( & & -0 , & & & & & 14 & 0 , & & 1,553 \\
\hline & 0 & & -0 & & 0 & & 0,564 & 17 & 0 , & & $\underline{1,563}$ \\
\hline 5 & -( & 922 & -0 , & 481 & & & & 43 & & & 1,614 \\
\hline 5647 & $-0,8$ & 3 & $-0,2$ & & & & 0, & 1934 & 0 & 44 & 1,626 \\
\hline 5 & $-0,80$ & & $-0,2$ & & & 2 & 0,5 & 5197 & 0,8 & 92 & 1,631 \\
\hline 5631 & $-0,7$ & & $-0,1$ & 3 & & 36 & 0,6 & 5342 & & 97 & 1,652 \\
\hline 560 & -0.77 & & $-0,1$ & & 0 & & $0, \mathrm{c}$ & 18 & 0, & 40 & 1,669 \\
\hline 55 & -06 & & -0 , & & 0 & 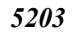 & 0,6 & 40 & 0 & 32 & 1,769 \\
\hline 4 & .633 & & -0 & 35 & 0,203 & 0 & 0 & 449 & 56 & $2 \underline{3}$ & ,995 \\
\hline 56 & 619 & & $-0,1 J 0$ & 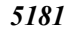 & & 26 & 0,617 & 334 & 0,969 & 13 & 2,236 \\
\hline 5632 & $-0,596$ & & $r$ & & & & 0,624 & 196 & & $\underline{48}$ & 2,282 \\
\hline 5070 &  & & ${ }^{\prime}, 1$ & & & & 0,636 & 28 & & 21 & 2,492 \\
\hline 5806 & $-0,5$ & & $-0,11$ & 7 & & $J_{1}$ & 0,641 & 31 & 0,979 & & $\underline{3,362}$ \\
\hline 5458 & $-0,555$ & 4820 & $-0,118$ & $50 \angle 0$ & 0,252 & 4720 & 0,651 & 5104 & 0,98 & $\underline{5336}$ & $\underline{4,84}$ \\
\hline
\end{tabular}




\section{Remarks}

This paper has dealt with early generation selection experiments in which various random or systematic arrangements of genotypes as well as various statistical techniques have been developed to increase the effectiveness and efficiencies of inference in such experiments. Usually the techniques are connected with spatial analysis. Because of certain limitations, mentioned earlier, it is often impossible to replicate the examined genotypes. Then it is necessary to adopt some assumptions concerning the spatial structure of the experimental material or to introduce into the experiment some plots with the same lines (very often varieties) called check plots. To the assumed correlation structure of experimental plots, geostatistical methods are applied (see for example Briggs and Shebeski, 1967; Zimmerman and Harville, 1991; Brownie and Gumpertz, 1997; Martin, 1986; Gołaszewski, 1999, 2002). In another approach, to recognize the spatial variability of the environment (soil), check plots are distributed over all experimental units. The problem is the density of the check plots and the way of using them in statistical analysis of the breeding experiments and in further inferences.

The literature contains many methods of using the information resulting from check plots. All of these methods have at least one weak point. They are developed for some specific proper structure of the environment, usually soil fertility. Their main disadvantage is the fact that before and also after the experiment we do not know what kind of the soil (environmental) structure is present in the experiment. Hence, we cannot say which of the existing methods is appropriate to a given experimental situation.

We have proposed here a method of using information from check plots that practically has no limitations on its use. In the proposed method we explore this information using a response surface methodology. Initially we try to identify the response surface characterizing the structure of an experimental environment. The obtained response surface is then used to adjust the 
observations for genotypes. Finally, the data so adjusted are used for statistical inference concerning selection for the next stages of the breeding program (ranking of genotypes).

\section{Acknowledgement}

This work was supported by the Polish National Science Centre (NCN) (grant no. N N310 111740)

\section{REFERENCES}

Baker R.J., McKenzie R.I.H. (1967): Use of control plots in yield trials. Crop. Sci. 7:335-337.

Box G.E.P., Draper N. (2007): Response Surfaces, Mixtures, and Ridge Analyses, Wiley, New York.

Bradley N. (2007): The response surface methodology. Indiana University South Bend.

Briggs K.G., Shebeski L.H. (1967): Implications concerning the frequency of control plots in wheat breeding nurseries. Can. J. Plant Sci. 48: 149 - 153.

Cullis B.R., Warwick J.A., Fisher B.J., Read B.J., Gleeson A.C. (1989): A new procedure for the analysis of early generation variety trials. Appl. Statist. 38: $361-375$.

Brownie C., Gumpertz M.L. (1997): Validity of Spatial Analyses for Field Trials. Journal of Agricultural, Biological, and Environmental Statistics 2(1): 1-23.

Gołaszewski J. (1999): Application of geostatistical methods to analysis of the data from a pea breeding trial. Biometrical Letters 36(2): 145-157.

Gołaszewski J. (2002): Geostatistical approach to data from field experiments with check plots - Electronic Journal of Polish Agricultural Universities, EJPAU, Ser. Agronomy 5(2).

Holtsmark G., Larsen B.R. (1905): Om multigheder for at indskraenke de fejl, som ved marksforsog betinges af jordens uensartethed. Tidsskrift for Landbrugets Planteavl, 12: 330-351.

Kempton R.A. (1984): The design and analysis of unreplicated field trials. Vortr. Pflanzenzuchtg 7: 219-242.

Kempton R.A., Fox P.N. (1997): Statistical methods for plant variety evaluation. Chapman \& Hall.

Khuri A.I., Cornell J.A. (1987): Response Surfaces. Designs and Analysis. Marcel Dekker, Inc. New York

Khuri A.I. (2006): Response Surface Methodology and Related Topics. World Scientific, Singapore.

Martin R.J. (1986): On the design of experiments under spatial correlation. Biometrika 73: $247-277$.

Mejza S., Marczyńska K. (2011): Check plot density in estimation of soil fertility. Proceedings: Biometric Methods and Models in Current Science and Research, D. 
Hampel, J. Hartmann, J. Michalek , (eds) Central Institute of Supervising and Testing in Agriculture, Brno: 167-172.

Myers an R.H., Montgomery D.C. (2001): Response Surface Methodology, John Wiley and Sons, 2nd edition.

Sebolai B., Pedersen J.F., Marx D.B., Boykin D.L. (2005): Effect of control plot density, control plot arrangements, and assumption of random or fixed effects on nonreplicated experiments for germplasm screening using spatial models. Crop Sciences: 1978-198.

Utz H.F. (1997): PLABSTAT. A computer program for statistical analysis of plant breeding experiments. Version 2N. Institute of Plant Breeding, Seed Science and Population Genetics. University of Hohenheim, Germany.

Zimmerman D.L., Harville D.A. (1991): A Random Field Approach to the Analysis of Field - Plot Experiments and Other Spatial Experiments. Biometrics 47: 223-239. 